\title{
Misidentification syndromes related to face specific area in the fusiform gyrus
}

\author{
Arthur J Hudson, Gloria M Grace
}

\begin{abstract}
The "delusional misidentification syndromes" are a group of uncommon and varied disorders in which, in typical form, the patient thinks that a particular familiar person is someone else or a certain familiar place is a duplicate. Although first identified and considered a memory disorder by Pick, evidence in support of this has been difficult to identify. They have been most often seen in various psychotic and organic brain diseases but lesions have been generally diffuse although the right temporal lobe has been implicated. A patient was investigated who abruptly developed a disorder wherein she misidentified her husband as her deceased sister and claimed that her home was a duplicate of her real home that were typical of Frégoli syndrome and Pick's reduplicative paramnesia, respectively. A discrete area of brain damage, probably ischaemic, in this patient was seen on MRI in the anterior part of the right fusiform gyrus and a smaller area in the nearby anterior middle and inferior temporal gyri with associated parahippocampal and hippocampal atrophy. A high order nervous system function that is devoted to the identification of faces is located in the adjacent midportion of the fusiform gyrus and a similar locus for environmental scenes, termed the parahippocampal place area, is present in the bordering parahippocampal gyrus. The misidentification phenomena in this case can be explained by disruption of the connections of these highly specialised areas with the most anterior inferior and medial part of the right temporal lobe where long term memory and mechanisms for the retrieval of information that are required for the visual recognition of faces and scenes are stored.

(F Neurol Neurosurg Psychiatry 2000;69:645-648)
\end{abstract}

Keywords: fusiform gyrus face area; parahippocampal place area; delusional misidentification; Frégoli syndrome

pondence

Dr A J Hudson

ahudson@julian.uwo.ca

Received 24 November 1999 and in final form

5 June 2000

Accepted 6 June 2000 consistently misidentifies a familiar place, sometimes including occupants, as a replica. Twenty years later a more common but similar phenomenon involving people, called "l'illusion des sosies" or Capgras syndrome, ${ }^{2}$ was described wherein an usually familiar person, such as a family member, is viewed as a stranger with an identical appearance. Since then, similar variants have been reported, including the Frégoli syndrome in which, as in the case described in this account, the patient conceptually misidentifies a person as another known person with an entirely different appearance. ${ }^{3}$ The cause of these delusional misidentification syndromes, as they are collectively known, ${ }^{4}$ is obscure although $\mathrm{Pick}^{1}$ maintained that the phenomenon represented a disturbance of memory, specifically involving "a sense of familiarity", that plays "an important part in remembrance, especially in recognition".

Although often described in relation to psychotic states including schizophrenia, it is, nevertheless, widely considered that these syndromes have an anatomical basis because of their frequent association with organic brain disease. ${ }^{45}$ Brain lesions generally have been poorly localised and are often bilateral although lesions in the right temporal lobe have been described. ${ }^{5}$ Sellal et $a l^{6}$ described both Capgras syndrome and delusional misidentification for place in a case of viral encephalitis with damage to the anterior medial part of the right temporal lobe and the medial frontal and orbital frontal regions bilaterally. Hirstein and Ramachandran ${ }^{7}$ postulated that Capgras syndrome could be explained by damage to connections from face processing areas in the right temporal lobe to the limbic system. Hence, our finding of discrete lesions in the right anterior inferior temporal lobe has provided a rare opportunity to confirm the suspected organic basis and anatomical localisation for these kinds of delusions. The visual phenomenon in these disorders is distinct from prosopagnosia in which familiar faces are perceived but not recognised. None the less, pure prosopagnosia is associated with damage to nearby right temporal cortical areas that include, predominantly, the lingual and posterior half of the fusiform and parahippocampal gyri. ${ }^{8} 9$ 


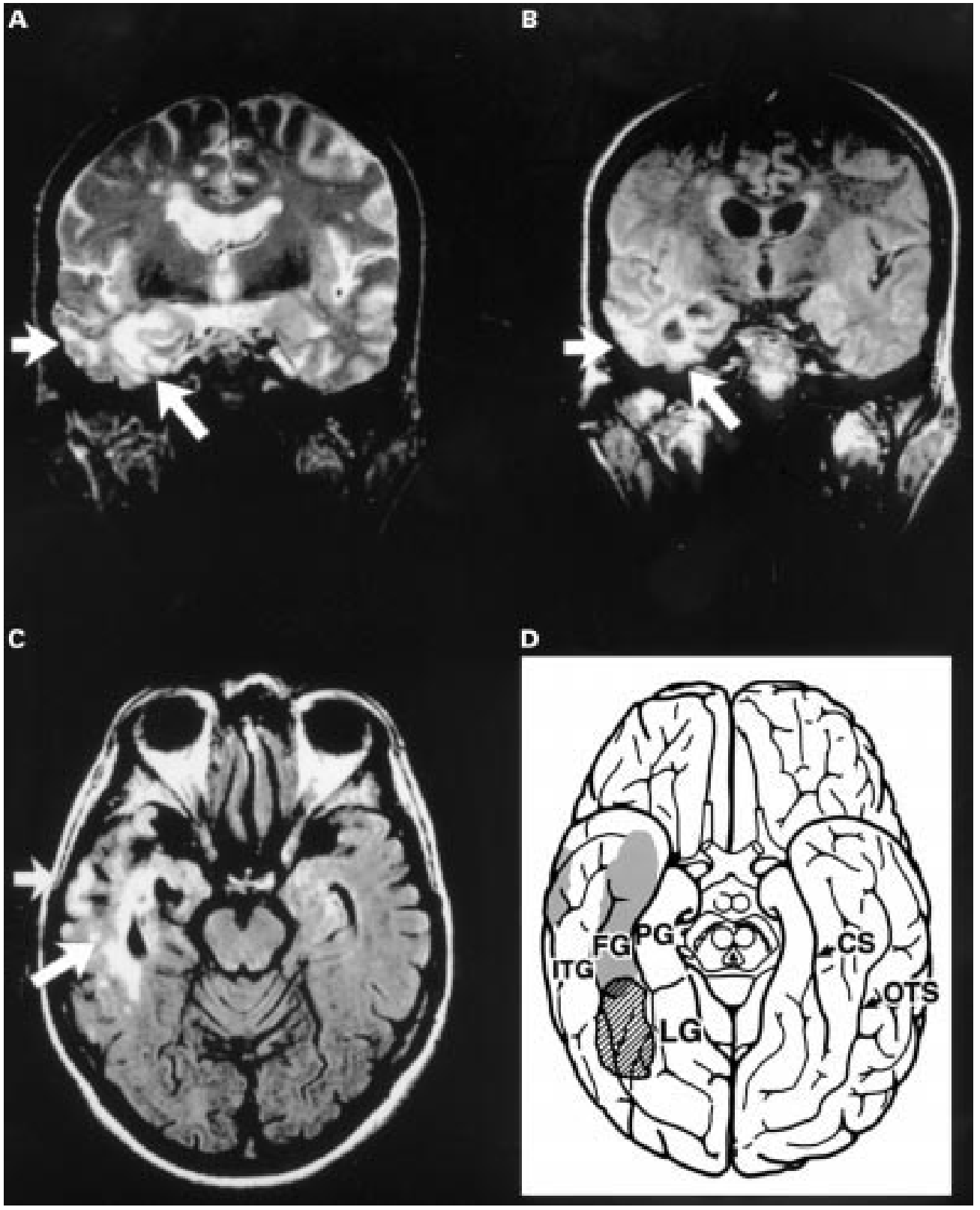

MRI of the patient's brain. (A) A coronal T2 weighted image through the temporal lobe at the level of the hippocampus shows abnormal hyperintensity in the right middle and inferior temporal gyrus (small arrow in all figures) and fusiform gyrus (large arrow in all figures). (B) Proton density image of the same area as shown in A, illustrates enlargement of the right temporal horn (divided by cut into two sections as in C). There are also atrophic changes in the right hippocampus and parahippocampal gyrus and (not shown) in the fusiform gyrus. (C) Axial FLAIR image through the temporal lobe at the level of the inferior tip of the temporal horn shows the anteroposterior extent of hyperintensity in the right inferior temporal and fusiform gyri. (D) Schematic illustration of the inferior surface of the brain demonstrates the approximate extent of the damage (grey shade) in the fusiform gyrus as derived from the MRI in comparison with the putative location and extent of the fusiform face (hatched) area. ${ }^{14} \mathrm{CS}=$ collateral sulcus; $F G=$ fusiform gyrus; $I T G=$ inferior temporal gyrus, $L G=$ lingual gyrus; $O T S=$ occipitotemporal sulcus; $P G=$ parahippocampal gyrus.

\section{Case report}

The subject, a 71 year old woman, began having frequent fainting attacks from intermittent complete heart block. This was confirmed when an electrocardiographic tracing obtained during an episode of unconsciousness disclosed an abrupt drop in her heart rate from 72 to 30 beats per minutes for 10 seconds. Within a day or two of the recording she was given a permanent cardiac pacemaker with immediate and complete arrest of fainting. However, during the first few days of the attacks, she began misidentifying her husband as her elder sister who had died 3 years previously. She would address him by the sister's name and would become argumentative and hostile towards him as though he were intruding. Intermittently she would speak to him using his real name, expressing their usual intimacy and clearly at such time recognising him as her husband. In this context it is noteworthy that visual contact seemed necessary for misidentification in as much as she never misidentified him as her sister on the telephone. In addition to misidentifying him she often referred to their home as a rented replica and would even pack her bags to return to their "real" home. Sometimes she thought other members of her family, all of 
Table 1 Neuropsychological tests

\begin{tabular}{|c|c|c|c|c|}
\hline \multirow[b]{3}{*}{ Test } & \multicolumn{4}{|c|}{ Classification range* } \\
\hline & \multicolumn{2}{|c|}{ Normal (average) } & \multicolumn{2}{|c|}{ Subnormal (impaired) } \\
\hline & High to mid & Low & Mild & Moderate to severe \\
\hline \multicolumn{5}{|l|}{ Wechsler adult intelligence scale-revised: } \\
\hline Verbal IQ & + & & & \\
\hline \multicolumn{5}{|l|}{ Subset scores } \\
\hline Information & + & & & \\
\hline Digit span & + & & & \\
\hline Vocabulary & + & & & \\
\hline Arithmetic & + & & & \\
\hline Comprehension & + & & & \\
\hline Similarities & + & & & \\
\hline Performance IQ & & + & & \\
\hline \multicolumn{5}{|l|}{ Subset scores: } \\
\hline Picture completion & & & + & \\
\hline Picture arrangement & & + & & \\
\hline Block design & & + & & \\
\hline Object assembly & & + & & \\
\hline Digit symbol & & + & & \\
\hline \multicolumn{5}{|l|}{ Wisconsin card sorting test: $†$} \\
\hline Number of categories & & + & & \\
\hline Perseverative responses & + & & & \\
\hline Non-perseverative responses & & & + & \\
\hline \multicolumn{5}{|l|}{ Trail making test: } \\
\hline Part A & & & & + \\
\hline Part B & & & & + \\
\hline \multicolumn{5}{|l|}{ Wide range achievement test -3 : } \\
\hline Reading subtest & + & & & \\
\hline \multicolumn{5}{|l|}{ Multilingual aphasia examination: } \\
\hline Benton visual naming & + & & & \\
\hline Controlled oral word association: & + & & & \\
\hline Semantic fluency test (animals) & & & & + \\
\hline \multicolumn{5}{|l|}{ Wechsler memory scale-revised: } \\
\hline Logical stories I & & + & & \\
\hline Logical stories II & + & & & \\
\hline Paired associate I & + & & & \\
\hline Paired associates II & + & & & \\
\hline Visual reproduction I & & & + & \\
\hline Visual reproduction II & & & + & \\
\hline Visual span & & & + & \\
\hline \multicolumn{5}{|l|}{ Rey auditory verbal learning test:† } \\
\hline Total words over 5 trials & & & + & \\
\hline List $B$ (words recalled) & & & & + \\
\hline Immediate recall list A & & & + & \\
\hline Delayed recall list A & & & + & \\
\hline Delayed recognition & & & + & \\
\hline \multicolumn{5}{|l|}{ Rey Osterrieth complex figure: $\dagger$} \\
\hline Copy & & & + & \\
\hline Delay & & & & + \\
\hline Clock drawing: $\dagger \ddagger$ & & & & + \\
\hline Oral and manual praxis screening $\ddagger$ & + & & & \\
\hline \multicolumn{5}{|l|}{ Grooved pegboard test: } \\
\hline Right hand & & & & + \\
\hline Left hand & & & + & \\
\hline Geriatric depression scale (items endorsed) & + & & & \\
\hline
\end{tabular}

${ }^{\star}$ Classification range based on age and/or education corrected normative data.

tQualitative evidence for perseveration and other executive dysfunction on these tests.

$\ddagger$ Evaluated qualitatively. temporal lobe (figure). There was also atrophy of the right parahippocampal and hippocampal gyri.

Performance on neuropsychological testing (table), about 1 year after the onset of the syndrome, was in the average to above average range on tests of verbal intellectual skill and reasoning, most language functions, mental calculation, auditory attention, and concentration (digit span), oral and manual praxis, and many aspects of verbal learning and memory. However, there were deficits in visual perception and construction and her performance IQ was significantly lower (25 points) than verbal IQ. Deficits were apparent in visual attention (visual span), memory for visual material (visual reproduction), and visual motor processing speed (trail making test). Executive processes such as word generation (semantic fluency) and organisational memory (Rey auditory verbal learning test) were mildly to moderately impaired, and several qualitative impairments of executive function were noted. For example, on delayed recall of the Rey complex figure, perseveration was illustrated when her drawing resembled not the appropriate figure but one drawn much earlier in the testing. Recognition of persons with whom she had fairly regular, but not intimate, contact and pictures of famous faces and places were markedly impaired when compared with control subjects although none was misidentified. By contrast, her identification of the faces of family members in old or relatively recent photographs, including those of herself, all siblings and her husband, was generally accurate, a disparity that is also seen in some cases of prosopagnosia. ${ }^{8}$ Moreover, she recognised herself in a mirror, and emotions displayed in photographs of faces were correctly interpreted. From a psychological perspective, misidentification of her husband as her sister and her home as a replica were alike in as much as they involved memory of familiar persons or scenes. Both types of delusions had in common the recall of specific remote memories of familiar persons or scenes that were evoked by visual contact with other familiar persons or scenes.

whom were either deceased or at the time living elsewhere, were occupying their house although when asked she could not locate them. Despite these relentless delusions, which were unchanged 2 years later, the patient was alert and cooperative and even openly discussed these experiences although insisting they were real. She seemed aware of her current surroundings during tests in hospital with no outward indication of thought disorders or other neurological abnormality. Speech was fluent and articulate, and affect and behaviour were appropriate. Epilepsy was excluded with three independent EEGs that showed no evidence of seizure activity and the delusional phenomena were not relieved by anticonvulsant medications. Brain MRI, performed 3 months after onset of delusions, showed damage, probably ischaemic in origin, to the anterior half of the right fusiform gyrus and a smaller area in the nearby middle and inferior gyri of the right

\section{Discussion}

The recent discovery of a face specific area in the fusiform gyrus ${ }^{11}{ }^{11}$ and a similar parahippocampal place area ${ }^{12}{ }^{13}$ within the same region as the damage in the anterior fusiform gyrus in this patient, has provided an opportunity to anatomically localise and consider how the visual misidentification syndromes were caused. The fusiform face area is located in the midportion of the right fusiform gyrus and, as shown in the figure, $\mathrm{D}$, lies immediately posterior to the damaged region. ${ }^{14}$ Thus, to relate the fusiform gyrus, it may be assumed that there has been interference with specific past visual memories causing impaired associations of human faces. Some possible anatomical connections of the fusiform face and parahippocampal place areas involving visual memory recall can be considered. Frégoli syndrome to the lesion in the anterior 
Sergent et al, ${ }^{15}$ using PET in face recognition tasks, found activation in the fusiform gyrus and the most anterior inferior part of the temporal lobe, including the temporal pole and the adjacent medial areas of both hemispheres. The right parahippocampal gyrus also showed increased activity. The anterior inferior temporal cortex has been described by Miyashita et $a l^{16-19}$ as the storehouse of long term visual memory where there are mechanisms for the retrieval and association of visually related information from the posterior cerebral cortices for the purpose of visual identification. Face recognition properties in the fusiform gyrus are considered to be among such memory related associative functions of the anterior inferior temporal region. ${ }^{18}$ However, the selection and retrieval of visual memory from this region, such as required for the recognition of human faces and scenes, is under the executive control of the prefrontal cortex. ${ }^{11} 1920$ Visual information encoded in, and retrieved from, long term memory also requires the participation of the parahippocampal cortex, which has a pivotal memory function relaying information between the hippocampus and cortex, including both the sensory association and prefrontal cortices. ${ }^{12} 18$ 20-22 Thus, the parahippocampal cortex may provide the means whereby stored memories, when related to current perceptions, are retrieved. ${ }^{18} 23$

The lesion in the right fusiform gyrus in this case extended from the face specific area in the middle of the gyrus to within a short distance from the tip of the temporal pole (figure, C and D). Consequently, the Frégoli syndrome is explained by damage having occurred to connections within the anterior fusiform gyrus that extend between the fusiform face area and the anterior inferior temporal association cortex on which appropriate retrieval of long term memory of faces would depend. ${ }^{10}$ Similarly, impairment of connections of the parahippocampal place specific area with the anterior inferior temporal cortex can account for the phenomenon in which the patient interpreted her house as a replica. ${ }^{132124}$ As found on psychological testing, prefrontal executive control of memory may also have been affected through injury to connections with the parahippocampal gyrus. However, a physiological explanation for the substitutive recall of remote visual memories in place of current experience, interpreted as "delusions", requires further knowledge of mnemonic processes.

We are grateful to Dr Donald Lee, Department of Diagnostic Radiology, Professor John A Kiernan, Department of Anatomy, Radiology, Professor John A Kiernan, Department of Anatomy, and Professor Tutis Vilis, Department of Physiology, University of Western Ontario, for their helpful suggestions in preparation
of the manuscript.

1 Pick A. Clinical studies. Brain 1903;26:242-67.

2 Capgras J, Reboul-Lachaux J. L'illusion des "sosies" dans un délire systématisé chronique. Bulletin de la Société Clinique Médécine Mentale 1923;2:6-16.

3 Courbon P, Fail G. Syndrome d' "illusion de Frégoli" et schizophrénie. Bulletin de la Société Clinique Médécine Menschizophrénie. Bulletin

4 Marková IS, Berrios GE. Delusional misidentifications: facts and fancies. Psychopathology 1994;27:136-43.

5 Feinberg TE, Shapiro RM. Misidentification: reduplication and the right hemisphere. Neuropsychiatry Neuropsychol Behav Neurol 1989;2:39-48.

6 Sellal F, Fontaine SF, Van Der Linden M, et al. To be or not to be at home? A neuropsychological approach to delusion for place. F Clin Exp Neuropsychol 1996;18:234-48.

7 Hirstein W, Ramachandran VS. Capgras syndrome: a novel probe for understanding the neural representation of the identity and familiarity of persons. Proc R Soc Lond B 1997; 264:437-44.

8 Damasio AR, Tranel D, Damasio H. Face agnosia and the neural substrates of memory. Annu Rev Neurosci 1990;13: $89-109$

9 Tranel D. Higher brain functions. In: Conn PM, ed. Neuroscience in medicine. Philadelphia: J B Lippincott, 1995:55580

10 McCarthy G, Puce A, Gore JC, et al. Face-specific processing in the human fusiform gyrus. $f \mathrm{Cogn}$ Neurosci 1997;9:605-10

11 Kanwisher N, McDermott J, Chun MM. The fusiform face area: a module in human extrastriate cortex specialized for face perception. F Neurosci 1997;17:4302-11.

12 Tong F, Nakayama K, Vaughan JT, et al. Binocular rivalry and visual awareness in human extrastriate cortex. Neuron 1998;21:753-9.

13 Epstein R, Kanwisher N. A cortical representation of the bcal visual environment. Nature 1998;392:598-601.

14 Tovée MJ. What are faces for? Curr Biol 1995;5:480-2.

15 Sergent J, Ohta S, Macdonald B. Functional neuroanatomy of face and object processing. A positron emission tomography study. Brain 1992;115:15-36.

16 Naya Y, Sakai K, Miyashita Y. Activity of primate inferotemporal neurons related to a sought target in pair-association task. Proc Natl Acad Sci USA 1996:93: 2664-9

17 Tomita H, Ohbayashi M, Nakahara K, et al. Top-down signal from prefrontal cortex in executive control of memory retrieval. Nature 1999;401:699-703.

18 Sakai K, Miyashita Y. Memory and imagery in the temporal lobe. Curr Opin Neurobiol 1993;3:166-70.

19 Hasegawa I, Fukushima T, Ihara T, et al. Callosal window between prefrontal cortices: cognitive interaction to retrieve long-term memory. Science 1998;281:814-18.

20 Brewer JB, Zhao Z, Desmond JE, et al. Making memories: brain activity that predicts how well visual experience will brain activity that predicts how well visual
be remembered. Science 1998;281:1185-7.

21 Van Hoesen GW. The parahippocampal gyrus. New observations regarding its cortical connections in the monkey. Trends Neurosci 1982;5:345-50.

22 Horton JC. The central visual pathways. In: Hart WM Jr, ed. Adler's physiology of the eye. St Louis: Mosby Year Book, 1992:728-72.

23 Goldman-Rakic PS, Selemon LD, Schwartz ML. Dual pathways connecting the dorsolateral prefrontal cortex with the hippocampal formation and parahippocampal
cortex in the rhesus monkey. Neuroscience 1984;12:719-43.

24 Teng E, Squire LR. Memory for places learned long ago is intact after hippocampal damage. Nature 1999;400:675-7. 